\title{
Enhancing Innovation through Knowledge Management: A Systematic Literature Review on Empirical Findings
}

\author{
Amelia Kurniawati, T. M. A. Ari Samadhi, Iwan I. Wiratmadja, Indryati Sunaryo, and Afrin F. Rizana
}

\begin{abstract}
To develop innovation initiative, an organization will highly rely and depends on the knowledge. By managing knowledge, an organization will increase its creativity that will bring positive impact on organizational performance and innovation. Since knowledge is considered as the determinant of innovation, this study is aimed to provide more comprehensive identification towards the relationship between knowledge management and innovation using systematic literature review on empirical findings. In the systematic literature review, 30 primary articles were obtained. According to 30 articles that were selected as the primary study, various knowledge management constructs, types of innovation, and mediating variable between knowledge management and innovation were identified.
\end{abstract}

Index Terms-Innovation, knowledge, knowledge management, systematic literature review.

\section{INTRODUCTION}

Innovation is defined as production or adoption, assimilation, and exploitation of a value-added novelty in economic and social spheres; renewal and enlargement of products, services, and markets; development of new methods of production; and the establishment of new management systems [1]. Firms with greater innovation capacity are considered greater in new product introduction and new market entry [2]. Moreover, innovation enables an organization to improve performance, solve problems, add value, and create the competitive advantage for the organization [3] and is considered as a key prerequisite for achieving sustained long-term wealth in today's business environment [4]. Thus, innovation is considered as necessary and critical components for an organization to create value, survive, and sustain competitive advantage [2], [5]. This need comes from increasing competition and customer demands, the emerging of new market areas [6] and rapid technological development [7]. To develop innovation initiative, an organization will highly rely and depends on the knowledge [2], [3]. Knowledge is considered as one of aspect that influences successful innovation [8]. By managing knowledge, an organization will increase its creativity that will bring positive impact on organizational performance and innovation [9]. Managing knowledge, basically involves the acquisition, creation, and use of information that can lead to

Manuscript received February 10, 2018; revised May 12, 2018.

Amelia Kurniawati is with Department of Industrial Engineering, Bandung Institute of Technology and Telkom University, Bandung, Indonesia (e-mail: amelia.kurniawati@gmail.com).

T. M. A. Ari Samadhi, Iwan Inrawan Wiratmadja, Indryati Sunaryo and Afrin Fauzya Rizana are with Department of Industrial Engineering, Bandung Institute of Technology, Bandung, Indonesia (e-mail: asamadhi@mail.ti.itb.ac.id, iwan@lspitb.ac.id, rya_ryo@yahoo.com, afrinfauzya2904@gmail.com0). innovation [10]. Therefore, knowledge management is considered as an effective means for increasing the innovation performance in an organization [11] and frequently cited as the antecedent of innovation [12]. Since knowledge management is considered as an important determinant of innovation, the comprehensive understanding towards the link between knowledge management and innovation is needed. An attempt to bring a clearer understanding of the linkage between these two constructs had been done by Costa \& Monteiro [13]. In their study, Costa \& Monteiro [13] identified the relationship between knowledge management process, namely knowledge acquisition, knowledge sharing, knowledge codification, and knowledge creation towards various type of innovation such as product/service and process innovation, radical and incremental innovation, technical and administrative innovation, innovation capability, and innovation performance. Other than that, the constructs in the study from Costa \& Monteiro [13] were obtained from 41 empirical articles and 4 theoretical articles. This study is aimed to provide more comprehensive identification towards the relationship between knowledge management and innovation using systematic literature review. The articles used as the primary study is limited to study that have empirical evidence regarding the relationship between knowledge management and innovation.

\section{MethodOLOGY}

This study aims to identify the comprehensive relationship of knowledge management on innovation. The systematic literature review protocols were adopted in this study to obtain the previous literature that discussed knowledge management and innovation. The literature review process followed five main steps in conducting the review on the study from Kitchenham \& Charters [14] namely, the identification of potential study, study selection, quality assessment, data extraction, and data analysis, as seen in Fig.1. In step 1, the potential literature to be reviewed were identified. It contains the process of initial search in the electronic database, internet, specific conference and journal, and any other sources. In this study, two electronic databases were used which are science direct and emerald insight. The search strategy was generated to obtain studies that only discuss specific issue of knowledge and innovation. The search was performed using Boolean AND towards the words "Knowledge" and "Innovation". In step 2, the literature that was obtained from step 1 was reviewed based on the specific criteria. There are four criteria used in this step. First, all of the studies must be written in the English language. Second, all of the studies must show and discuss 
the relationship between knowledge and innovation. Third, the unit analysis is an organization, and finally, the literature obtained should perform empirical evidence towards the relationship between knowledge and innovation. In step 3, the literature that passes step 2 will be further reviewed to check its quality. In step 4, the primary studies were selected. Finally, in step 5, the primary studies were further review and analyzed.

\section{RESULT AND DISCUSSION}

The literature review process in this study is presented in Fig. 2. The number of articles obtained from Science Direct and Emerald Insight database in initial search based on the abstract is 4216 and 936 respectively. The further identification of the appropriate articles that were obtained in the previous step was performed. From 936 articles found in Emerald Insight, 861 articles were excluded. Those articles were removed because the title of the articles did not specifically mention or discuss knowledge and innovation. Using the same reason, 3802 articles form Science Direct were also excluded for further reviewed.

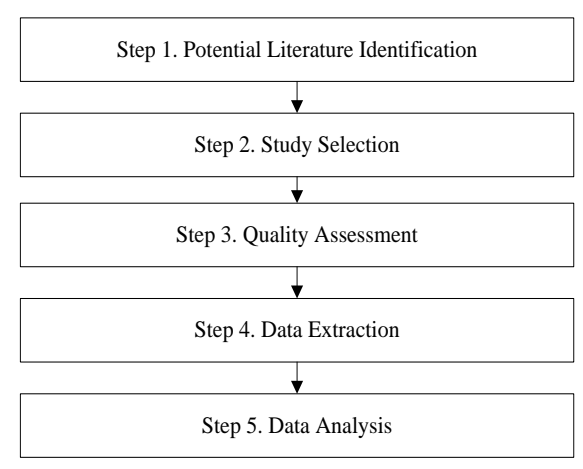

Fig. 1. Systematic literature review steps.

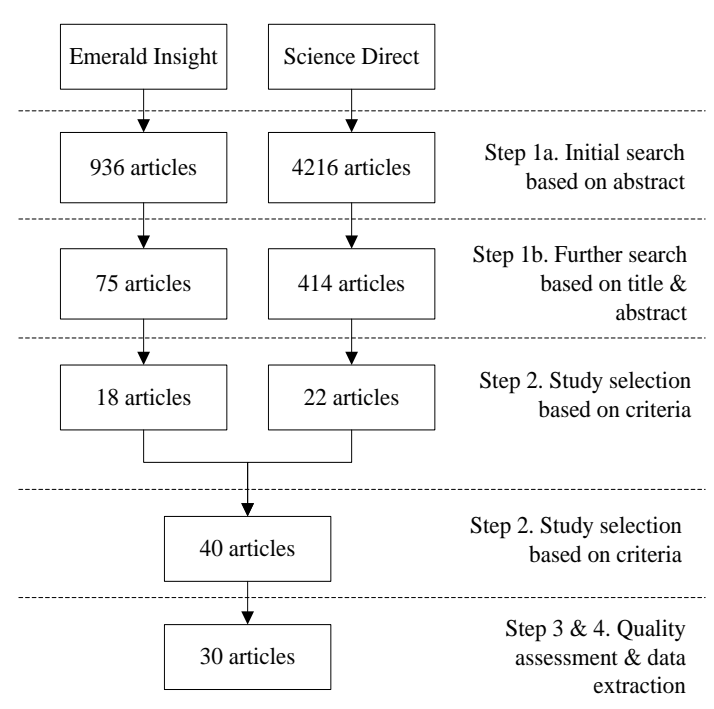

Fig. 2. Literature review process.

The remaining 75 and 414 articles from Emerald Insight and Science Direct were examined whether they met the criteria or not based on the existence of relationship between knowledge and innovation, empirical evidence of the relationship, whether they were written using English language or not, and the unit analysis of the study must be in organizational level. From 75 remaining articles in Emerald Insight, only 18 articles that met all the criteria, while in Science Direct only 22 articles that met all the criteria. Therefore, there are 40 articles in total from both databases to be further reviewed and screened to obtain primary articles. Finally, 30 articles were selected as primary articles to be analyzed in this study. According to 30 articles that were selected as the primary study, various knowledge management constructs, types of innovation, and mediating variable between knowledge management and innovation were identified. The summary of the primary articles obtained in this study can be seen in Table I.

\section{A. Knowledge Management}

There are three categories of knowledge management construct obtained in this study, namely knowledge management process and capabilities, knowledge management implementation approach, and knowledge characteristic. Knowledge management process and capabilities consist of knowledge exploration, knowledge acquisition, knowledge creation, knowledge dissemination, knowledge storage, and knowledge application. In addition to knowledge management capabilities, Darroch [15] considered responsiveness to knowledge as a construct that measures knowledge management capabilities. The term of knowledge management process and capabilities that was between one primary article to another article might differ. Knowledge acquisition construct has similar meaning with knowledge collecting and knowledge exploitation. Knowledge dissemination construct has similar meaning with knowledge sharing, knowledge transfer, knowledge distribution, and knowledge donating. Knowledge creation is considered has similar meaning with knowledge conversion. Knowledge application is considered has similar meaning with knowledge interpretation and knowledge utilization.

The next knowledge management category is knowledge management implementation approach [16]. According to López-Nicolás \& Meroño-Cerdán [17], there are two types of knowledge management implementation approach, namely personalization and codification, while Obeidat, Al-Suradi, Masa'deh, and Tarhini [16], identified three approaches, namely personalization, codification, and social network.

The last knowledge management category is knowledge characteristic. There are four knowledge categories identified, which are knowledge complexity [18], [19], knowledge tacitness [18]. Knowledge explicit [19], and modularity [19].

\section{B. Innovation}

This study identified various types of innovation variables to measure the existence of innovation improvement in an organization. Innovation variables used in one article might differ with another article. Innovation can be measured in different ways depending on the interest of the researcher [10]. According to literature study, there are 12 types of innovation that are related to knowledge management. 
TABLE I: THE SUMmaRY OF PRIMARY ARTICLES

\begin{tabular}{|c|c|c|c|c|}
\hline Reference & Object & KM Manifest & Innovation Manifest & Proven Relationship \\
\hline [20] & $\begin{array}{l}\text { companies operating } \\
\text { within the district of this } \\
\text { city }\end{array}$ & $\begin{array}{l}\text {-Knowledge collecting } \\
\text {-knowledge donating }\end{array}$ & $\begin{array}{l}\text { - Innovation capability } \\
\text { - Innovation } \\
\text { performance } \\
\end{array}$ & $\begin{array}{l}\text {-knowledge donation --> innovation capability } \\
\text {-innovation capability --> innovation } \\
\text { performance }\end{array}$ \\
\hline$[21]$ & 3 organization in Turkey & $\begin{array}{l}\text { - knowledge donating } \\
\text { - knowledge collecting } \\
\end{array}$ & Innovation Capability & - knowledge collecting --> innovation capability \\
\hline$[22]$ & Service firm in Bahrain & $\begin{array}{l}\text { - Knowledge acquisition } \\
\text { - knowledge transfer } \\
\text { - knowledge application }\end{array}$ & $\begin{array}{l}\text { - product innovation } \\
\text { - process innovation }\end{array}$ & $\begin{array}{l}\text { - KM acquisition --> process innovation } \\
\text { - Knowledge transfer --> process innovation } \\
\text { - knowledge application --> process \& product } \\
\text { innovation }\end{array}$ \\
\hline [23] & $\begin{array}{l}\text { three main kinds of parks } \\
\text { that make up the industrial } \\
\text { cluster index in Taiwan }\end{array}$ & $\begin{array}{l}\text { - knowledge creation \& } \\
\text { acquisition } \\
\text { - knowledge dissemination } \\
\text { \& storage }\end{array}$ & $\begin{array}{l}\text { - Market innovation } \\
\text { performance } \\
\text { - product innovation } \\
\text { performance }\end{array}$ & $\begin{array}{l}\text { - KM --> Innovation } \\
\text { - Knowledge creation \& acquisition --> } \\
\text { Innovation } \\
\text { - knowledge dissemination \& storage --> } \\
\text { Innovation }\end{array}$ \\
\hline [24] & Spanish Industrial Firms & $\begin{array}{l}\text {-Internal knowledge } \\
\text { creation capacity } \\
\text { - Absorptive capacity }\end{array}$ & $\begin{array}{l}\text {-Incremental } \\
\text { innovation } \\
\text { - Radical Innovation }\end{array}$ & $\begin{array}{l}\text { - internal knowledge creation capacity --> } \\
\text { incremental innovation } \\
\text { - Absorptive capacity --> Incremental } \\
\text { Innovation } \\
\text { - Absorptive capacity --> Radical Innovation } \\
\text { - internal knowledge creation capacity --> } \\
\text { absorptive capacity } \\
\text { - absorptive capacity mediated internal } \\
\text { knowledge creation and radical innovation } \\
\end{array}$ \\
\hline [25] & $\begin{array}{l}4 \text { mobile } \\
\text { telecommunication } \\
\text { companies }\end{array}$ & Knowledge Sharing & $\begin{array}{l}\text { Innovation } \\
\text { performance }\end{array}$ & $\begin{array}{l}\text {-Innovation Technology Capability --> } \\
\text { Innovation } \\
\text { - Innovation technology Support --> Innovation } \\
\text { - Knowledge Sharing --> Innovation } \\
\text { - Innovation Technology Capability --> } \\
\text { Knowledge Sharing } \\
\text { - Innovation technology Support --> Knowledge } \\
\text { Sharing }\end{array}$ \\
\hline [26] & $\begin{array}{l}\text { Online Technology firms } \\
\text { (Weibo, Wechat, Linkedin, } \\
\text { etc) }\end{array}$ & "Knowledge Acquisition & "-Innovation strategy & - Knowledge acquisition --> innovation \\
\hline [27] & "Technology firms & $\begin{array}{l}\text {-KM Transfer } \\
\text { - KM Storage } \\
\text { - KM Application } \\
\text {-KM Creation } \\
\end{array}$ & $\begin{array}{l}\text { - Innovation } \\
\text { performance }\end{array}$ & $\begin{array}{l}\text { - KM creation --> innovation } \\
\text { - KM application practice --> innovation }\end{array}$ \\
\hline [28] & $\begin{array}{l}\text { Business units in } \\
\text { Taiwanese Firms }\end{array}$ & $\begin{array}{l}\text { - Knowledge acquisition } \\
\text { capability } \\
\text { - knowledge sharing } \\
\text { capability } \\
\end{array}$ & Radical Innovation & $\begin{array}{l}\text {-Knowledge acquisition capabilities --> radical } \\
\text { innovation } \\
\text { - Knowledge sharing capabilities --> radical } \\
\text { innovation } \\
\end{array}$ \\
\hline [29] & Italian Firms & $\begin{array}{l}\text { - Knowledge management } \\
\text { system } \\
\text { - Knowledge management } \\
\text { capacity } \\
\end{array}$ & $\begin{array}{l}\text { - Open Innovation } \\
\text { - Innovation Capacity }\end{array}$ & $\begin{array}{l}\text { - KMS --> KMC through open innovation } \\
\text { - Open Innovation --> KM Capacity } \\
\text { - Open innovation --> innovation capacity } \\
\text { - KMC --> Innovation capacity } \\
\end{array}$ \\
\hline [18] & "Manufacturing firms & $\begin{array}{l}\text { - Knowledge tacitness } \\
\text { - Knowledge complexity }\end{array}$ & - Radical Innovation & $\begin{array}{l}\text { - Social capital; --> Radical innovation } \\
\text { - Knowledge complexity --> Radical Innovation } \\
\text { - Knowledge tacitness --> Radical Innovation } \\
\end{array}$ \\
\hline [17] & $\begin{array}{l}\text { 310 Organization in } \\
\text { Spanish }\end{array}$ & $\begin{array}{l}\text { - Codification KM strategy } \\
\text { - Personalisation KM } \\
\text { Strategy }\end{array}$ & - Radical Innovation & $\begin{array}{l}\text { - Codification KM Strategy --> Innovation } \\
\text { - Personalisation KM Strategy -->Innovation }\end{array}$ \\
\hline [30] & 150 firms & $\begin{array}{l}\text {-knowledge management } \\
\text { capacity (acquisition, } \\
\text { sharing, application) }\end{array}$ & $\begin{array}{l}\text {-innovation } \\
\text { performance }\end{array}$ & $\begin{array}{l}\text {-knowledge management capacity --> firm } \\
\text { innovation performance }\end{array}$ \\
\hline [31] & $\begin{array}{l}\text { Manufacturing \& Financial } \\
\text { firms }\end{array}$ & $\begin{array}{l}\text { Organizational Capabilities } \\
\text { - Knowledge acquisition } \\
\text { - Knowledge conversion } \\
\text { - Knowledge Application }\end{array}$ & $\begin{array}{l}\text { Organizational } \\
\text { Innovation }\end{array}$ & $\begin{array}{l}\text { - Knowledge management --> Organizational } \\
\text { Innovation } \\
\text { - Knowledge management --> organizational } \\
\text { learning } \\
\text { - Organizational learning --> Organizational } \\
\text { innovation } \\
\end{array}$ \\
\hline$[32]$ & 218 project by 144 firms & - Knowledge Acquisition & - Product Innovation & - Knowledge Acquisition --> Product Innovation \\
\hline [33] & firms in Turkey & $\begin{array}{l}\text { - Knowledge management } \\
\text { skill }\end{array}$ & $\begin{array}{l}\text { - Innovation } \\
\text { performance }\end{array}$ & - KM Skill --> Innovation performance \\
\hline [34] & "Manufacturing Firms & $\begin{array}{l}\text { Knowledge } \\
\text { Management Process } \\
\text { - Knowledge acquisition } \\
\text { - Knowledge sharing } \\
\text { - Knowledge application } \\
\end{array}$ & $\begin{array}{l}\text { - Product } \\
\text { Innovation } \\
\text { - Process Innovation }\end{array}$ & $\begin{array}{l}\text { - KM Process --> Product Innovation } \\
\text { - KM Process --> Process Innovation }\end{array}$ \\
\hline [35] & Manufacturing SME & $\begin{array}{l}\text { - Knowledge } \\
\text { management capability }\end{array}$ & - Open Innovation & - KM Capabilities --> Open Innovation \\
\hline
\end{tabular}


TABLE I: THE SUMMARY OF PRIMARY ARTICLES (CONTINUED)

\begin{tabular}{|c|c|c|c|c|}
\hline Reference & Object & KM Manifest & Innovation Manifest & Proven Relationship \\
\hline [36] & Manufacturing firms & $\begin{array}{l}\text { - Knowledge acquisition } \\
\text { - Knowledge sharing } \\
\text { - Knowledge application } \\
\text { - Knowledge storage }\end{array}$ & $\begin{array}{l}\text { - Technological } \\
\text { Innovation }\end{array}$ & $\begin{array}{l}\text { - Knowledge sharing --> Technological } \\
\text { Innovation } \\
\text { - Knowledge Storage --> Technological } \\
\text { Innovation } \\
\text { - Knowledge Application --> Technological } \\
\text { Innovation } \\
\text { - Knowledge Acquisition --> knowledge } \\
\text { application } \\
\text { - Knowledge Acquisition --> Knowledge sharing } \\
\text { - Knowledge sharing --> knowledge application }\end{array}$ \\
\hline [37] & High tech firms in Taiwan & $\begin{array}{l}\text { Cust. Knowledge } \\
\text { management } \\
\text { - Knowledge acquisition } \\
\text { - Knowledge Sharing } \\
\text { - Knowledge Application }\end{array}$ & $\begin{array}{l}\text { - Product Innovation } \\
\text { Performance }\end{array}$ & $\begin{array}{l}\text { - Cust Knowledge Management --> Product } \\
\text { Innovation Performance }\end{array}$ \\
\hline [7] & $\begin{array}{l}\text { Malaysian Manufacturing } \\
\text { Sector }\end{array}$ & $\begin{array}{l}\text { KM Process } \\
\text { - Knowledge Acquisition } \\
\text { - Knowledge dissemination } \\
\text { - Knowledge application }\end{array}$ & $\begin{array}{l}\text { - Innovation } \\
\text { performance }\end{array}$ & $\begin{array}{l}\text { - KM Process --> Innovation } \\
\text { - Knowledge Application --> Innovation } \\
\text { - Knowledge dissemination --> Innovation }\end{array}$ \\
\hline [38] & 111 Spanish companies & $\begin{array}{l}\text { - Knowledge exploration } \\
\text { - Knowledge exploitation }\end{array}$ & & $\begin{array}{l}\text { - Knowledge exploration --> Innovation } \\
\text { - Knowledge exploitation --> Innovation }\end{array}$ \\
\hline [2] & 176 firms in Taiwan & $\begin{array}{l}\text { - Knowledge acquisition } \\
\text { - Knowledge sharing } \\
\text { - Knowledge application }\end{array}$ & $\begin{array}{l}\text { Innovation } \\
\text { - Administrative } \\
\text { Innovation } \\
\text { - Technical } \\
\text { Innovation }\end{array}$ & $\begin{array}{l}\text { - All of the mediators are proven } \\
\text { - Knowledge management --> Innovation }\end{array}$ \\
\hline [12] & Spanish Companies & $\begin{array}{l}\text { - Knowledge Acquisition } \\
\text { - Knowledge distribution } \\
\text { - Knowledge Interpretation }\end{array}$ & $\begin{array}{l}\text { - Product innovation } \\
\text { - Process innovation }\end{array}$ & $\begin{array}{l}\text { - KM --> Product Innovation } \\
\text { - KM --> Process Innovation }\end{array}$ \\
\hline [19] & KM Managers & $\begin{array}{l}\text { KM Capability } \\
\text { - Acquisition } \\
\text { - Conversion } \\
\text { - Application } \\
\text { Knowledge Characteristic } \\
\text { - Modularity } \\
\text { - Complexity } \\
\text { - Explicity } \\
\text { Knowledge Integration } \\
\text { - Efficiency } \\
\text { - Flexibility }\end{array}$ & $\begin{array}{l}\text { - Product innovation } \\
\text { - Process innovation }\end{array}$ & $\begin{array}{l}\text { - Knowledge integration --> Innovation } \\
\text { - K Char --> KM Capability } \\
\text { - KM Capabilities --> Innovation } \\
\text { - Org Learning --> Innovation }\end{array}$ \\
\hline [15] & $\begin{array}{l}\text { CEO from cross-section } \\
\text { Industries in New Zealand }\end{array}$ & $\begin{array}{l}\text { - knowledge acquisition } \\
\text { - Knowledge dissemination } \\
\text { - knowledge } \\
\text { responsiveness }\end{array}$ & Innovation & $\begin{array}{l}\text { - Knowledge acquisition --> Innovation } \\
\text { - Knowledge dissemination --> innovation } \\
\text { - Knowledge responsiveness --> Innovation }\end{array}$ \\
\hline [39] & Manufacturing Company & - Knowledge management & $\begin{array}{l}\text { - Technical } \\
\text { Innovation }\end{array}$ & $\begin{array}{l}\text { - Knowledge management --> technical } \\
\text { innovation }\end{array}$ \\
\hline [16] & $\begin{array}{l}\text { Jordanian Consulting } \\
\text { Firms }\end{array}$ & $\begin{array}{l}\text { Knowledge management } \\
\text { process } \\
\text { - Acquisition } \\
\text { - Sharing } \\
\text { - Utilization } \\
\text { KM Approach } \\
\text { - Social network } \\
\text { - Codification } \\
\text { - Personalization }\end{array}$ & Innovation & $\begin{array}{l}\text { - KM Process (Acquisition, sharing, utilization) } \\
\text {--> Innovation } \\
\text { - KM Approach (codification, social network, } \\
\text { personalization) --> Innovation }\end{array}$ \\
\hline [40] & French Firms & $\begin{array}{l}\text { Knowledge management } \\
\text { - Knowledge sharing } \\
\text { - Knowledge capture and } \\
\text { acquisition } \\
\text { - Policies and Strategy } \\
\text { - Training and Mentoring }\end{array}$ & $\begin{array}{l}\text { environmental } \\
\text { innovation }\end{array}$ & - KM --> Environmental innovation \\
\hline$[10]$ & $\begin{array}{l}\text { Parastatals Organization in } \\
\text { Uganda }\end{array}$ & Knowledge management & $\begin{array}{l}\text { Organizational } \\
\text { innovation }\end{array}$ & - KM --> Organizational Innovation \\
\hline
\end{tabular}

\section{Innovation Performance}

In this study, eight articles used innovation performance variable to empirically test the relationship between knowledge management and innovation. Innovation performance was defined as the performance of the implementation new and improved method in the terms of marketing, business practice, workplace organization or external relation, and also the implementation of the new and improved product (goods or service) or process [20].

According to the literature review, knowledge exploration, acquisition, creation, sharing, storage, application, responsiveness to knowledge, and knowledge management implementation approach, as listed in Table II, are related to innovation performance.

As an addition, the study of Kamasak, Yavuz, Karagulle, \& Agca [33] provides empiric evidence that knowledge management skill has positive relationship towards innovation performance, where it can be argued that 
knowledge management skill is similar to knowledge management capability. Knowledge management capability consists of capability in knowledge management processes such as knowledge exploration, acquisition, creation, sharing, storage, and application.

TABLE II: KM AND INNOVATION PERFORMANCE

\begin{tabular}{ll}
\hline \hline $\begin{array}{l}\text { Knowledge management variable from } \\
\text { literature }\end{array}$ & Reference \\
\hline $\begin{array}{l}\text { Knowledge exploration } \\
\text { Knowledge acquisition }\end{array}$ & {$[38]$} \\
Knowledge creation & {$[15],[16],[20],[30],[38]$} \\
Knowledge dissemination & {$[27]$} \\
Knowledge storage & {$[15],[16],[20],[25],[30]$,} \\
Knowledge Application & {$[27]$} \\
Responsiveness to knowledge & {$[16],[27],[30]$} \\
Knowledge management implementation & {$[15]$} \\
approach & {$[16]$} \\
\hline
\end{tabular}

\section{Product and Process Innovation}

Based on primary articles used in this study, seven articles used either process innovation, product innovation, or both process and product innovation variable to empirically test the relationship between knowledge management and innovation. Product innovation is related to both introducing new products and improving existing ones [34]. It consists of the introduction of a new-to-the-world product, product modification and improvement, as well as the production of the product that is new to the customer through the extending the existing product line in the organization [22]. Process innovation is defined as the introduction of new changes in the way of producing the product or service to improve efficiency [22] through the adoption of new or improved methods [12]. The knowledge management variables that positively influence product and process innovation can be seen in Table III.

TABLE III: KM AND PRODUCT \& PROCESS INNOVATION

\begin{tabular}{lll}
\hline \hline $\begin{array}{l}\text { Knowledge management } \\
\text { variable from literature }\end{array}$ & Innovation Type & Reference \\
\hline Knowledge creation & $\begin{array}{l}\text { Product Innovation } \\
\text { Process Innovation }\end{array}$ & {$[19],[23]$} \\
& P19] \\
Knowledge acquisition & Product Innovation & {$[23],[32],[34]$,} \\
& Process Innovation & {$[37],[12],[19]$} \\
Knowledge dissemination & Product Innovation & {$[34],[37],[19]$} \\
& Process Innovation & {$[22],[12]$} \\
Knowledge Storage & Product Innovation & {$[23]$} \\
& Process Innovation & {$[23]$} \\
Knowledge Application & Product Innovation & {$[22],[34],[37]$} \\
& & {$[12],[19]$} \\
& Process Innovation & {$[22],[12],[19]$} \\
\hline \hline
\end{tabular}

\section{E. Market Innovation}

Based on primary articles used in this study, there is only one article that used market innovation variable to empirically test the relationship between knowledge management and innovation. Market innovation is defined as the performance of the organization related to high customer's demand, satisfaction, the increasing market share, and profit [23]. Moreover, according to the empirical study, knowledge creation and acquisition, and knowledge storage and dissemination influence innovation performance including market innovation [23].

\section{F. Incremental and Radical Innovation}

Based on primary articles used in this study, four articles used radical innovation, incremental innovation, or both radical and incremental innovation variable to empirically test the relationship between knowledge management and innovation. Incremental innovation consists of the refinement and reinforcement of current products, processes, technologies, methods, and organizational structure in the organization, while radical innovation related the innovation that produces fundamental changes towards products, processes, technologies, methods, or organizational structure in the organization [24]. The knowledge management variables that positively influence radical and incremental innovation are presented in Table IV.

TABLE IV: KM AND RADICAL \& INCREMENTAL INNOVATION

\begin{tabular}{|c|c|c|}
\hline $\begin{array}{l}\text { Knowledge management } \\
\text { variable from literature }\end{array}$ & Innovation Type & Reference \\
\hline Knowledge creation & Incremental Innovation & {$[24]$} \\
\hline \multirow[t]{2}{*}{ Knowledge acquisition } & Radical Innovation & {$[24],[28]$} \\
\hline & Incremental Innovation & {$[24]$} \\
\hline Knowledge dissemination & Radical Innovation & {$[24],[28]$} \\
\hline Knowledge application & Radical Innovation & {$[24]$} \\
\hline \multicolumn{3}{|l|}{ Knowledge Characteristic } \\
\hline - $\quad$ Tacitness & Radical Innovation & {$[18]$} \\
\hline - $\quad$ Complexity & Radical Innovation & {$[18]$} \\
\hline \multicolumn{3}{|l|}{$\begin{array}{l}\text { Knowledge } \\
\text { Implementation Approach }\end{array}$} \\
\hline Codification & Radical Innovation & [17] \\
\hline - $\quad$ Personalization & Radical Innovation & [17] \\
\hline
\end{tabular}

\section{G. Administrative and Technical Innovation}

Three articles used administrative innovation, technical innovation, or both administrative and technical innovation variable to empirically test the relationship between knowledge management and innovation. Administrative innovation measure the innovation in planning procedure, process control, and integrated mechanism [2]. Technical or technological innovation is defined as the innovation that encompasses innovation in products and processes [39]. Ayoub, Abdallah, \& Suifan [39] proved the relationship between knowledge management in general, which is represented by knowledge acquisition, dissemination, and application, toward technical innovation, but not specifically breakdown knowledge management variable and test each dimension towards innovation. The knowledge management variables that positively influence administrative and technical innovation are listed in Table V.

TABLE V: KM AND ADMINISTRATIVE \&TECHNICAL INNOVATION

\begin{tabular}{lll}
\hline \hline $\begin{array}{l}\text { Knowledge management } \\
\text { variable from literature }\end{array}$ & Innovation Type & Reference \\
\hline Knowledge acquisition & $\begin{array}{l}\text { Administrative Innovation } \\
\text { Technical Innovation }\end{array}$ & {$[2]$} \\
& Ad6], [2] & {$[2]$} \\
Knowledge dissemination & Technical Innovation & {$[36],[2]$} \\
Knowledge storage & Technical Innovation & {$[36]$} \\
Knowledge application & Administrative Innovation & {$[2]$} \\
& Technical Innovation & {$[36],[2]$} \\
\hline \hline
\end{tabular}




\section{H. Open Innovation}

Based on primary articles used in this study, there are two articles that used open innovation variable to empirically test the relationship between knowledge management and innovation. Open innovation is an innovation where organization tends to build up collaborations with the external partner to access and benefit from their new technologies, skills, and expertise [35]. Knowledge management capability has positive a relationship with open innovation [29], [35].

\section{Organizational Innovation}

Based on primary articles used in this study, two articles used organizational innovation variable to empirically test the relationship between knowledge management and innovation. Organizational innovation is defined as the application of the new idea in the organization, where it is embodied in products, processes, and management marketing system [31]. Both articles provide empirical evidence of the relationship of knowledge management in general towards organizational innovation. Mafabi, Muene, \& Ntayi [10] consider knowledge management as a system that consists of knowledge capture and acquisition, creation, dissemination, and storage where Liao and $\mathrm{Wu}$ [31] consider knowledge acquisition, knowledge creation, and knowledge application as the dimension of knowledge management.

\section{J. Environmental Innovation}

Based on primary articles used in this study, only one article used environmental innovation variable to empirically test the relationship between knowledge management and innovation [26]. Environmental or green innovation has been defined as the new or modified practices, methods, system, and products that decrease environmental harm through the replacement of inefficient business activities [26]. Stanovcic, Pekovic, \& Bouziri [26] examine three constructs of knowledge management, namely knowledge management in general (including knowledge capture and acquisition, knowledge sharing or dissemination, policies and strategy, and training and mentoring), knowledge management policies, and knowledge sharing culture. All three constructs have positive relationship towards environmental innovation.

\section{K. Mediation Variable}

According to the literature review, there are some articles that analyze the mediation effect. Below is the further explanation regarding the mediation effect obtained from primary articles. According to the study developed by Yeşil, Koska, \& Büyükbeşe [20], knowledge donating, which in this study is referred to knowledge dissemination have an indirect effect towards innovation performance. Innovation capability mediates the effect of knowledge dissemination towards innovation performance. The study from Forez \& Camison [24], found that the effect of knowledge creation towards radical innovation was mediated by absorptive capacity.

\section{CONCLUSION}

In conclusion, this study performed a systematic literature review to obtain more insight regarding the relationship between knowledge and innovation. There are thirty articles were selected as primary articles for further review. According to the primary articles, there are three categories of knowledge management that influence innovation, namely knowledge management process, knowledge management implementation approach, and knowledge characteristic. Moreover, various types of innovation were also obtained namely, innovation performance, product and process innovation, technical and administrative innovation, radical and incremental innovation, market innovation, open innovation, and organizational innovation. For further research, the effect of knowledge management on innovation might be categorized based on the category of the organization such as SME, the non-profit organization, government, etc. Moreover, according to this literature study, most of the innovation still limited to closed innovation that utilizes internal resource which is difficult to be implemented in small industries due to the limited resource. Therefore, small industries tend to adopt collaborative or open innovation method that enables the use of the external resource. The role of knowledge management in open innovation needs further investigation because it is possible that open innovation requires both intra-organizational and inter-organizational knowledge management.

\section{REFERENCES}

[1] Crossan and M. Apaydin, "A multi-dimensional framework of organizational innovation: A systematic review of the literature," Journal of Management Studies, vol. 47, no. 6, pp. 1154-1191, 2010.

[2] J. Huang and Y. Li, "The mediating effect of knowledge management on social interaction and innovation performance," International Journal of Manpower, vol. 30, no. 3, pp. 285-301, 2009.

[3] M. Gloet and M. Terziovski, "Exploring the relationship between knowledge management practices and innovation performance," Journal of Manufacturing Technology Management, vol. 15, no. 5, pp. 402-409, 2004.

[4] D Esterhuizen, C. S. L. Schutte, and A. S. A du Toit, "Knowledge creation processes as critical enablers for innovation," International Journal of Information Management, vol. 32, pp. 354-364, 2012.

[5] M. Yavarzadeh, Y. Salamzadeh, and A. Poormokhtari Ardakani, "Investigating the role of knowledge management in organizational innovation and its effect on organization's performance. Case study: Tax affairs general administration of Yazd province," International Journal of Management Sciences and Business Research, vol. 4, no. 9 , pp. 50-67, 2015.

[6] R. McAdam, "Knowledge management as a catalyst for Innovation within organizations: A qualitative study," Knowledge and Process Management, vol. 7, no. 4, pp. 233-241, 2000.

[7] M. Yusr, S. Sanuri Mohd Mokhtar, A. Rahim Othman, and Y. Sulaiman, "Does interaction between TQM practices and knowledge management processes enhance the innovation performance?" International Journal of Quality \& Reliability Management, vol. 34, no. 7, pp. 955-974, 2017.

[8] S. Roper, H. Dundas, and Nola, "Knowledge stocks, knowledge flows and innovation: Evidence from matched patents and innovation panel data," Research Policy, vol. 44, pp. 1327-1340, 2015.

[9] H. Lee and B. Choi, "Knowledge management enablers, processes, and organizational performance: An integrative view and empirical examination," Journal of Management Information Systems, pp. 179228, 2003.

[10]S. Mafabi, J. Muene, and J. Ntayi, "Knowledge management and organisational resilience: Organisational innovation as a mediator in Uganda parastatals," Journal of Strategy and Management, vol. 5, no. 1, pp. 57-80, 2012.

[11]H. Tapio Inken, A. Kianto, and M. Vanhala, "Knowledge management practices and innovation performance in Finland," Baltic Journal of Management, vol. 10, no. 4, pp. 432-455, 2015.

[12]D. Jiménez-Jiménez and R. Sanz-Valle, "Managing human resources in order to promote knowledge management and technical innovation," Management Research: Journal of the Iberoamerican Academy of Management, vol. 5, no. 2, pp. 83-100, 2007. 
[13] V. Consta and S. Monteiro, "Key knowledge management processes for innovation: a systematic literature review," VINE Journal of Information and Knowledge Management Systems, vol. 46, no. 3, 2016

[14]B. Kitchenham and S. Charters, Guidelines for performing Systematic Literature Reviews in Software Engineering (Technical Report No. EBSE-2007-01). Evidence-Based Software Engineering, Keele, UK: Keele University, 2007, pp. 5-65.

[15]J. Darroch, "Knowledge management, innovation and firm performance," Journal of Knowledge Management, vol. 9, no. 3, pp. 101-115, 2005.

[16] B. Yousef Obeidat, M. Al-Suradi, R. Masa'deh, and T. Ali, "The impact of knowledge management on innovation: an empirical study on Jordanian consultancy firms," Management Research Review, vol. 39, no. $10,2016$.

[17]C. López-Nicolás and A. L. Mero no-Cerdán, "Strategic knowledge management, innovation and performance," International Journal of Information Management, vol. 31, pp. 502-509, 2011.

[18] A. Pérez-Luño, C. Cabello Medina, A. Carmona Lavado, and G. Cuevas Rodríguez, "How social capital and knowledge affect innovation," Journal of Business Research, 2011.

[19] T. Ju, C. Li, and T. Lee, "A contingency model for knowledge management capability and innovation," Industrial Management \& Data, vol. 106, no. 6, pp. 855-877, 2006.

[20] S. Yeşil, A. Koska, and T.Büyükbeşe, "Knowledge sharing process, innovation capability and innovation performance: An empirical study," in Proc. 2nd International Conference on Leadership, Technology and Innovation Management, 2013.

[21]S. Yeşil and S. Ferit Dereli, "An empirical investigation of the organisational justice, knowledge sharing and innovation capability," in Proc. 2nd International Conference on Leadership, Technology and Innovation Management, 2013.

[22]M. Birasnav, M. Albufalasa, and Y. Bader, "The role of transformational leadership and knowledge management processes on predicting product and process innovation: An empirical study developed in Kingdom of Bahrain," TÉKHNE - Review of Applied Management Studies, vol. 11, pp. 64-75, 2013.

[23] Y. Lai, M. Hsu, F. Lin, Y. Chen, and Yi-Hsin Lin, "The effects of industry cluster knowledge management on innovation performance," Journal of Business Research, vol. 67, 2014.

[24]B. Forés and C. Camisón, "Does incremental and radical innovation performance depend on different types of knowledge accumulation capabilities and organizational size?" Journal of Business Research, 2016.

[25] N. Ismaeel and J. Qammach, "The mediating role of knowledge sharing on relationship between IT capability and IT support as predictors of innovation performance: An empirical study on mobile companies in Iraq," in Proc. 3rd Global Conference on Business, Economics, Management, and Tourism, 2016.

[26] B. Nguyen, X. Yu, T. C. Melewar, and J. Chen, "Brand innovation and social media: Knowledge acquisition from social media, market orientation, and the moderating role of social media strategic capability," Industrial Marketing Management, 2015.

[27]M. J. Donate and J. D. Sánchez de Pablo, "The role of knowledge-oriented leadership in knowledge management practices and innovation," Journal of Business Research, 2014.

[28] C. C. J. Cheng, C.Yang, and C. Sheu, "Effects of open innovation and knowledge-based dynamic capabilities on radical innovation: An empirical study," Journal of Engineering and Technology Management vol. 41, pp. 79-91, 2016

[29] Indre Maurer, "How to build trust in inter-organizational projects: The impact of project staffing and project rewards on the formation of trust, knowledge acquisition and product innovation," International Journal of Project Management, vol. 28, pp. 629-637, 2010.

[30]C. Chen and J. Huang, "Strategic human resource practices and innovation performance - The mediating role of knowledge management capacity," Journal of Business Research, vol. 62, pp. 104-114, 2009.

[31] S. Liao and C. Wu, "System perspective of knowledge management, organizational learning, and organizational innovation," Expert System with Application, vol. 37, pp. 1096-1103, 2010

[32] G. Santoro, D. Vrontis, A.Thrassou, and L. Dezi, "The internet of things: Building a knowledge management system for open innovation and knowledge management capacity," Technological Forecasting \& Social Change, 2017.

[33] R. Kamasak, M. Yavuz, A. Ozgur Karagulle, and T. Agca, "Importance of strategic flexibility on the knowledge and innovation relationship: An Emerging Market Study," in Proc. 5th International Conference on Leadership, Technology, Innovation and Business Management.

[34] A. F. Al-Sa'di, A. B. Abdallah, A. B. Abdallah, S. E. Dahiyat, and S. E. Dahiyat, "The mediating role of product and process innovations on the relationship between," Business Process Management Journal, vol. 23, 2017.

[35] I. Martinez-Conesa, P. Soto-Acosta, and E. George Carayannis, "On the path towards open innovation: Assessing the role of knowledge management capability and environmental dynamism in SMEs," Journal of Knowledge Management, vol. 21, no. 3, 2017.

[36] V. Lee, L. Leong, T. Hew, and K. Ooi, “Knowledge management: A key determinant in advancing technological innovation?" Journal of Knowledge Management, vol. 17, no. 6, pp. 848-872, 2013.

[37] R. Lin, R. Che, and C. Ting, "Turning knowledge management into innovation in the high tech industry," Industrial Management \& Data Systems, vol. 112, no. 1, pp. 42-63, 2012.

[38] M. Javier Donate and F. Guadamillas, "Organizational factors to support knowledge management and innovation," Journal of Knowledge Management, vol. 15, no. 6, pp. 890-914, 2011.

[39] H.Fawzi Ayoub, A. Bahjat Abdallah, and T. S. Suifan, "The effect of supply chain integration technical innovation in Jordan: The mediating role of knowledge management," Benchmarking: An International Journal, vol. 24, no. 3, 2017.

[40] T. Stanovcic, S. Pekovic, and A. Bouziri, "The effect of knowledge management on environmental innovation," Baltic Journal of Management, vol. 10, no. 4, 2015.

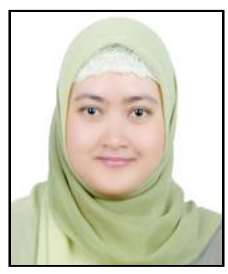

Amelia Kurniawati is a lecturer at Department of Industrial Engineering, Telkom University in Bandung, Indonesia. She received Master Degree from Bandung Institute of Technology Indonesia in 2009. She is now a Ph.D Student at Bandung Institute of Technology Indonesia. Her current research interest is in the area of knowledge management and organizational performance.

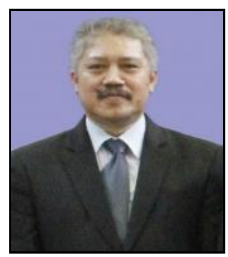

TMA Ari Samadhi is an associate professor at Department of Industrial Engineering, Bandung Institute of Technology in Bandung, Indonesia. He received Doctoral Degree from University of New South Wales, Australia in Production Network field. His current research interest is in technology and manufacturing system.

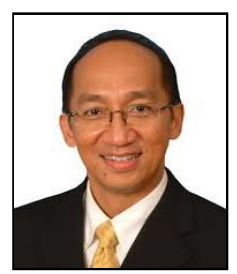

Iwan Inrawan Wiratmadja is an associate professor at Department of Industrial Engineering, Bandung Institute of Technology in Bandung, Indonesia. He received Doctoral Degree from Ecole Superieure des Affaire-University Pierre Mendes France, Grenoble in 1995 in the management of technology field. His current research interest is in technology and knowledge management.

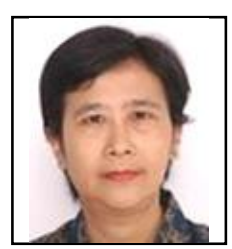

Indryati Sunaryo is an associate professor at Department of Industrial Engineering, Bandung Institute of Technology in Bandung, Indonesia. She received Doctoral Degree from Bandung Institute of Technology, Indonesia in 2005. Her current research interest is in Human Resource Management.

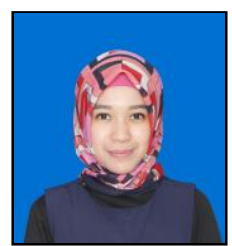

Afrin Fauzya Rizana is a lecturer in Department of Industrial Engineering, Telkom University, Bandung, Indonesia. She receives her master degree in Industrial Engineering and Management from Bandung Institute of Technology Indonesia in 2016. Her current research interest is in information system management and knowledge management. 